6 N. Beverini, P. Minguzzi u. F. Strumia, Phys. Rev. A 4, 350 [1971].

7 W. Happer, Rev. Mod. Phys. 44, 169 [1972].

8 S. J. Davis, J. J. Wright u. L. C. Balling, Phys. Rev. A 3, 1220 [1971].

9 A. Assfalg, J. Fricke, J. Haas, R. Tilgner u. E. Lüscher, Z. Naturforsch. 26 a, 1567 [1971].

10 R. Tilgner, J. Fricke u. J. Haas, Helv. Phys. Acta 42, 337 [1969].

11 M. A. Bouchiat, J. Phys. Radium 24, 379 [1963] ; 24, 611 [1963].

12 M. A. Bouchiat, Publ. Sci. Techn. Ministère de L'Air, Nr. N. T. 146, Paris [1965].

13 B. R. Bulos u. W. Happer, Phys. Rev. A 4, 849 [1971].

14 C. C. Bouchiat, M. A. Bouchiat u. L. C. Pottier, Phys. Rev. 181, 144 [1969].

15 M. A. Bouchiat, J. Brossel u. C. Pottier, J. Chem. Phys. 56, 3703 [1972]
16 V. A. Komarovskit, N. P. Penkin u. L. N. Shabanova, Opt. Spectr. 25, 81 [1968].

17 E. U. Condon u. G. H. Shortley, The Theory of Atomic Spectra, Cambridge Univ. Press, Cambridge 1964.

18 J. Kunl, Z. Physik 242, 66 [1971].

19 J. E. Hansen, A. Steudel u. H. Walther, Z. Phys. 203, 296 [1967].

20 A. Assfalg, J. Fricke, J. Haas u. E. Lüscher, Z. Naturforsch. 26 a, 1571 [1971].

21 J. K. Mitchell u. E. N. Fortson, Phys. Rev. Lett. 28, 5 [1972].

22 W. Franzen, Phys. Rev. 115, 850 [1959].

23 P. Minguzzi, F. Strumia u. P. Violino, Nuovo Cim. 46 B, 145 [1966].

24 L. C. Balling, R. M. Lambert, J. J. Wright u. R. E. Weiss, Phys. Rev. Lett. 22, 161 [1969].

25 E. A. Mason u. T. R. Marrero, Advances in Atomic and Molecular Physics 6, 155, London 1970.

26 P. Violino, Suppl. Nuovo Cim. 6, 440 [1968].

\title{
Hochtemperatur-Mikrowellenspektrometer für Zeeman-Effekt-Messungen an diamagnetischen Molekeln
}

\author{
$g_{J}$-Faktor von TIF, CsF, CsCl, CsBr, CsI und Anisotropie der magnetischen Suszeptibilität von TlF, CsF \\ und $\mathrm{CsCl}$
}

\section{R. HONERJÄGER und R. TISCHER}

II. Physikalisches Institut der Freien Universität Berlin, D 1 Berlin 33, Boltzmannstraße 20

(Z. Naturfor-ch. 28 a, $458-463$ [1973] ; eingegangen am 17. Dezember 1972)

A High-Temperature Microwave Spectrometer for Measurements of Zeeman Effect in diamagnetic Molecules. - $g_{J}$-Factor of TlF, CsF, CsCl, CsBr, CsI and Magnetic Susceptibility Anisotropy of $\mathrm{TlF}, \mathrm{CsF}$ and $\mathrm{CsCl}$

\begin{abstract}
A high-temperature microwave spectrometer has been developed for Zeeman effect measurements in strong magnetic fields up to $50 \mathrm{kG}$. The absorption cell is placed in a superconducting solenoid and can be heated as high as $1200^{\circ} \mathrm{C}$. The $g_{J}$-factor and the magnetic susceptibility anisotropy of $\mathrm{TlF}, \mathrm{CsF}$ and $\mathrm{CsCl}$, the $g_{J}$-factor of $\mathrm{CsBr}$, and an upper limit for the value of the $g_{J}$-factor of $\mathrm{CsI}$ have been measured. The molecules $\mathrm{CsF}$ and $\mathrm{CsCl}$ were also studied in higher vibrational states.
\end{abstract}

Zur Untersuchung des Rotations-Zeeman-Effektes diamagnetischer Molekeln werden im wesentlichen zwei Typen von hochauflösenden Spektrometern benutzt: elektrische und magnetische Molekularstrahlresonanz-Apparaturen und Mikrowellenspektrometer mit einer Absorptionszelle im Magnetfeld. ZeemanEffekt-Messungen an kleinen anorganischen Molekeln, die durch Verdampfen der entsprechenden festen Stoffe erst bei höherer Temperatur in ausreichender Konzentration entstehen, sind bislang nur mit Spektrometern vom Molekularstrahl-Typ durchgeführt worden. Die Zahl der auf diese Weise untersuchten Molekeln ist gering. In dieser Arbeit wird ein einfaches und weitgehend universell verwend-

Sonderdruckanforderungen an Prof. Dr. R. HONERJÄGER, D-1000 Berlin 45, Karwendelstr. 27 c. bares Mikrowellenspektrometer mit heizbarer Absorptionszelle beschrieben, welches für Zeeman-Effekt-Messungen in starken Magnetfeldern geeignet ist. Messungen am TlF und den Cs-Halogeniden dienten in erster Linie der Erprobung des neuen Spektrometers. Einige dieser Molekeln sind bereits von GRÄFF u. a. ${ }^{1}$ (TIF, CsF) und RAmsey u. a. ${ }^{2}$ $(\mathrm{CsF}, \mathrm{CsCl})$ mit der Methode der elektrischen bzw. magnetischen Molekularstrahlresonanz untersucht worden, so daß unsere Meßergebnisse mit ihren verglichen werden können.

Die wesentlichen Elemente der heizbaren Absorptionszelle sind in Abb. 1 a und $1 \mathrm{~b}$ dargestellt. Die Absorptionszelle des im übrigen konventionellen Mikrowellenspektrometers mit Stark-Effekt-Modulation besteht aus Tantalblech von $0,1 \mathrm{~mm}$ Dicke, wel- 
wassergekühlter Vakuummantel

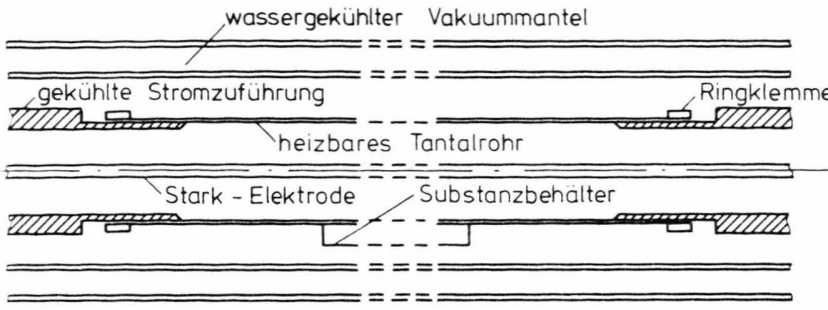

Abb. 1 a. Achsschnitt durch die Absorptionszelle.

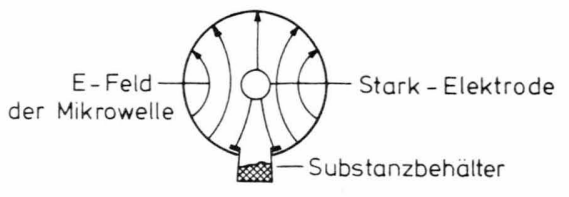

Abb. 1 b. Querschnitt durch das Tantalrohr mit $5 \mathrm{~mm}$ breitem Längsschlitz und eingehängtem Substanzbehälter. Das Mikrowellen-E્E-Feld der angeregten $H_{11}$-Welle verläuft ganz in der Querschnittsebene.

ches bis auf einen verbleibenden Längsschlitz von $5 \mathrm{~mm}$ Breite zu einem kreiszylindrischen Rohr von $17 \mathrm{~mm}$ Durchmesser und $280 \mathrm{~mm}$ Länge gebogen ist. Es wird direkt durch Stromfluß $(50 \mathrm{~Hz}$ Wechselstrom) erhitzt. Für eine Temperatur von $600^{\circ} \mathrm{C}$ werden etwa $70 \mathrm{~A}$ (bei ca. $100 \mathrm{~W}$ ) benötigt, für $1200{ }^{\circ} \mathrm{C}$ etwa $180 \mathrm{~A}$ (bei ca. $1 \mathrm{~kW}$ ). Im Schlitz hängt der aus Tantalblech geformte, oben offene Substanzbehälter. Die beiderseits anschließende, ebenfalls kreiszylindrische Mikrowellenleitung dient als Stromzuführung. Sie wird in der Nähe der heißen Zelle gekühlt. Die Temperatur der Zelle - und damit der Dampfdruck der erhitzten Substanz - ist über einen mittleren Bereich von $L=180 \mathrm{~mm}$ Länge annähernd konstant; $L$ stellt also auch die ungefähre wirksame Absorptionsstrecke dar. Die koaxiale Stark-Elektrode besteht aus einem Edelstahlrohr von $3 \mathrm{~mm}$ Durchmesser; ein Keramikstab im Rohrinnern verhütet ein Durchbiegen des Rohres beim Erwärmen. Die heizbare Zelle nebst einem Teil der beiderseits anschließenden Mikrowellenleitung befindet sich innerhalb eines evakuierbaren, kreiszylindrischen und wassergekühlten Messingrohers. Das Innere des Tantalrohres wird ausschließlich durch den nicht abgedeckten Teil des Längsschlitzes evakuiert.

Die gesamte Zellenanordnung hat einen äußeren Durchmesser von $50 \mathrm{~mm}$ und läßt sich zentriert in die horizontale Raumtemperatur-Bohrung (60 mm Durchmesser) eines supraleitenden Magneten einfahren. Der Kryomagnet vom U-Boot-Typ (Hersteller: The Oxford Instrument Company Ltd., Oxford, England) enthält ein vierteiliges Solenoid (Niob-
Titan-Multifilamentleiter) von insgesamt $280 \mathrm{~mm}$ Länge und an den Enden erhöhter Wicklungsdichte. Die maximal erreichbare Feldstärke beträgt $50 \mathrm{kG}$. Das Feld ist über den Absorptionsraum (Kreiszylinder von $180 \mathrm{~mm}$ Länge und $17 \mathrm{~mm}$ Durchmesser) auf $1 \%$ homogen. Magnetfeld und Heizstrom durch die Mikrowellenabsorptionszelle verlaufen also weitgehend parallel zueinander. Die Erfahrung hat gezeigt, daß Lorentz-Kräfte auf die stromdurchflossene Zelle im Magnetfeld bis zu den höchsten Feldstärken so klein bleiben, daß Störungen durch Verformungen der Zelle nicht auftreten. Im Absorptionsraum stehen Magnetfeld und elektrischer Vektor eines linear polarisierten Mikrowellenfeldes vom $H_{11}$-Typ senkrecht aufeinander; d.h. in dieser Anordnung werden $\sigma$-Zeeman-Linien beobachtet. Messung mil einer um das Magnetfeld zirkular polarisierten $H_{11}$. Welle erlaubt, das.Vorzeichen von $g_{J}$ zu bestimmen.

Die Magnetfeldstärke wird durch den Solenoidstrom gemessen. Geeicht wurde bei verschiedenen Feldstärken (bis zu $25 \mathrm{kG}$ ) mittels der Elektronenspinresonanz von gepulvertem Diphenylpicrylhydrazyl (DPPH) (isotroper $g$-Faktor: 2,00366). Bei einem Meßfehler von $10^{-3}$ fanden wir keine Abweichung von der Proportionalität zwischen der Stromstärke durch das Solenoid und der magnetischen Feldstärke. Wegen der Kleinheit der DPPH-Sonde konnten wir den Verlauf des $H$-Feldes längs der Solenoidachse „punktweise“ ausmessen. Innerhalb der Fehlergrenzen stimmen die Meßwerte mit den aus der Solenoidgeometrie berechneten Daten überein. Bei der Auswertung einer zur Feldstärke $H$ proportionalen Linienaufspaltung wird der über die Absorptionsstrecke gemittelte $H$-Wert benutzt; er ist um $4,6 \%$ größer als im Solenoidmittelpunkt.

Die Frequenz der $\sigma$-Zeeman-Komponenten $(m \rightarrow m \pm 1)$ von Rotationslinien $(J \rightarrow J+1)$ einer zweiatomigen Molekel mit den Kernspins $I_{1}=I_{2}=0$ beträgt ${ }^{3}$

$$
\begin{aligned}
v_{ \pm}(J, m, H) & =v_{0}(J) \mp g_{J}\left(\mu_{n} / h\right) H \\
& -\left(\xi_{T} / h\right) f^{ \pm}(J, m) H^{2} .
\end{aligned}
$$

Oberes (unteres) Vorzeichen gilt für den Übergang $m \rightarrow m+1 \quad(m \rightarrow m-1)$. Es bedeuten $H$ das angelegte Magnetfeld, $h$ die Plancksche Konstante, $g_{J}$ das molekulare gyromagnetische Verhältnis relativ zum Kernmagneton $\mu_{n}, \quad \xi_{T}=(1 / 3)\left(\xi_{\perp}-\xi_{\|}\right)$die Anisotropie der Magnetisierbarkeit der Molekel $\left(\xi_{\perp}\right.$ und $\xi_{\|}$sind die Magnetisierbarkeiten senkrecht bzw. 
parallel zur Kernverbindungslinie) und

$f^{ \pm}(J, m)=\frac{3(m \pm 1)^{2}-(J+1)(J+2)}{(2 J+1)(2 J+5)}-\frac{3 m^{2}-J(J+1)}{(2 J-1)(2 J+3)}$

Eine Rotationslinie der Frequenz $v_{0}(J)$ spaltet im Magnetfeld in $2(2 J+1) \sigma$-Komponenten auf, die wegen des Gliedes mit $\xi_{T}$ in charakteristischer Weise unsymmetrisch zur feldfreien Linie $v_{0}$ liegen. In Abb. 2 ist das nach Gl. (1) berechnete Spektrum

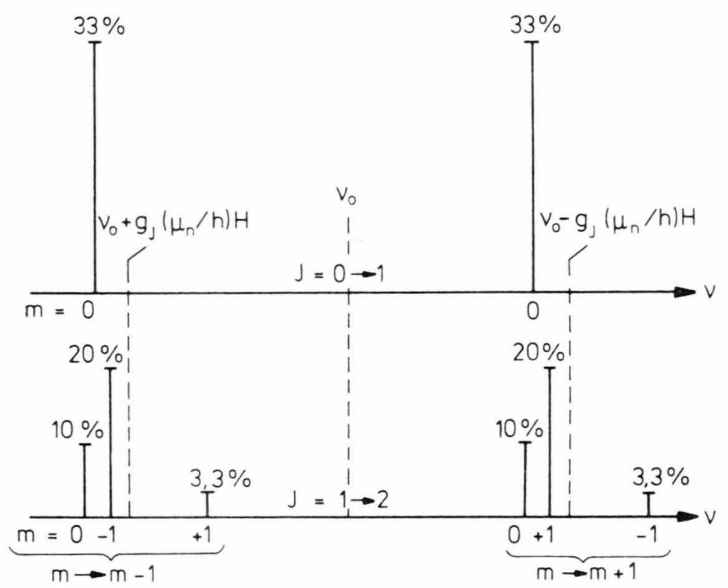

Abb. 2. Intensität und charakteristische Lage der $\sigma$-ZeemanKomponenten im Falle $g_{J}<0$ und $\xi_{T}>0$.

der $\sigma$-Komponenten für die beiden tiefsten Rotationsübergänge $J=0 \rightarrow 1$ und $J=1 \rightarrow 2$ dargestellt. Die Linienintensitäten $I^{ \pm}(J, m)$ bezogen auf die Intensität $I_{0}(J)$ der nicht aufgespaltenen Rotationslinie sind berechnet nach ${ }^{4}$

$$
\frac{I^{ \pm}(J, m)}{I_{0}(J)}=\frac{(J+2 \pm m)(J+1 \pm m)}{2(J+1)(2 J+1)(2 J+3)} .
$$

Es gilt

$$
\sum_{m=-J}^{+J} I^{+}(J, m)=\sum_{m=-J}^{+J} I^{-}(J, m)=(1 / 3) I_{0}(J) .
$$

Die charakteristische Verteilung von Lage und Intensität der Linien über das Spektrum ist Folge der Relationen

$$
\begin{aligned}
& f^{-}(J, m)=f^{+}(J,-m), \\
& I^{-}(J, m)=I^{+}(J,-m) .
\end{aligned}
$$

Diejenigen Linien, die infolge der Anisotropie der Magnetisierbarkeit im Magnetfeld am meisten verschoben werden und deshalb am besten zur Messung von $\xi_{T} / h$ geeignet sind, haben leider die kleinste relative Intensität.
Wir betrachten fortan einen festen Rotationsübergang $J \rightarrow J+1$ und unterdrücken $J$ überall dort, wo dies unmißverständlich möglich ist. Zunächst werde angenommen, daß zumindest einzelne Paare „korrespondierender" Linien $m \rightarrow m+1$ und $-m \rightarrow-m-1$ vom Spektrometer aufgelöst, ihre Frequenzen $v_{+}(m, H)$ bzw. $v_{-}(-m, H)$ also meßbar sind. Aus den Gln. (1) und (5) folgt der Frequenzabstand

$v_{+}(m, H)-v_{-}(-m, H)=-2 g_{J}\left(\mu_{n} / h\right) H$.

Er ist unabhängig von $m$ und $\xi_{T}$. Aus den gemessenen Frequenzen $v_{+}$und $v_{-}$bei unterschiedlichen Feldstärken (und gegebenenfalls verschiedenen Rotationsübergängen) wird durch Ausgleich und Mittelung ein Bestwert $\bar{g}_{J}$ gewonnen. Mit diesem Bestwert für $g_{J}$ erhält man aus Gl. (1)

$$
v_{ \pm}(m, H) \pm \bar{g}_{J}\left(\mu_{n} / h\right) H=v_{0}-\left(\xi_{T} / h\right) f^{ \pm}(m) H^{2} .
$$

Zur Auswertung nach $\xi_{T}$ werden die „Meßwerte“ $v_{ \pm} \pm \bar{g}_{J}\left(\mu_{n} / h\right) H$ nach der Methode der kleinsten Fehlerquadrate an den theoretischen Kurvenverlauf $v_{0}-\left(\xi_{T} / h\right) f^{ \pm}(m) H^{2}$ angepaßt.

Bei den in dieser Arbeit untersuchten Molekeln beobachtet man auch für Übergänge $(J \geqq 1 \rightarrow J+1)$ bis zum höchsten hier benutzten Magnetfeld eine Aufspaltung der Rotationslinien in nur zwei „ $\sigma$-Linien “: $\xi_{T}$ ist so klein, daß bei der experimentell vorgegebenen Linienbreite die Aufspaltung nach $m$ nur noch zum Teil aufgelöst wird ${ }^{5}$. Von den $2 J+1$ $\sigma$-Komponenten der Gruppe $m \rightarrow m+1$ erscheinen die intensitätsstärkeren als eine mehr oder minder asymmetrische "Linie“, während die restlichen zwar aufgelöst sind, aber infolge zu geringer Intensität unbeobachtbar bleiben. Das gleiche gilt entsprechend für die $\sigma$-Komponenten der Gruppe $m \rightarrow m-1$. In diesem Falle wird auf folgende Weise nach $g_{J}$ und $\xi_{T}$ ausgewertet. $I_{v}^{+}(v, H)$ sei die beim Felde $H$ registrierte spektrale Intensität der Liniengruppe $m \rightarrow m+1$ :

$$
\int_{v=0}^{\infty} I_{v}^{+}(v, H) \mathrm{d} v=\sum_{m=-J}^{+J} I^{+}(m) .
$$

Unter der Annahme, daß die $2 J+1$ Einzellinien dieser Liniengruppe Lorentz-Form und gleiche Linienbreite $(2 \Delta v=$ volle Halbwertsbreite) haben, erhält man

$I_{v}{ }^{+}(v, H)=\sum_{m=-J}^{+J} \frac{I^{+}(m)}{\pi} \frac{\Delta v}{\left[v-v_{+}(m, H)\right]^{2}+(\Delta v)^{2}}$, 
somit

$$
\begin{aligned}
I_{v}^{+} & {\left[v-g_{J}\left(\mu_{n} / h\right) H, H\right] } \\
& =\sum_{m=-J}^{+J} \frac{I^{+}(m)}{\pi} \frac{\Delta v}{\left[v-v_{0}+\left(\xi_{T} / h\right) f^{+}(m) H^{2}\right]^{2}+(\Delta v)^{2}}
\end{aligned}
$$

und entsprechend

$$
\begin{aligned}
I_{v}{ }^{-}[v & \left.+g_{J}\left(\mu_{n} / h\right) H, H\right] \\
& =\sum_{m=-J}^{+J} \frac{I^{-}(m)}{\pi} \frac{\Delta v}{\left[v-v_{0}+\left(\xi_{T} / h\right) f^{-}(m) H^{2}\right]^{2}+(\Delta v)^{2}} .
\end{aligned}
$$

Mit Rücksicht auf die Relationen (5) und (6) folgt

$I_{v}{ }^{+}\left[v-g_{J}\left(\mu_{n} / h\right) H, H\right] \equiv I_{v}{ }^{-}\left[v+g_{J}\left(\mu_{n} / h\right) H, H\right]$,

d. h. beide Linienprofile werden durch Wechselwirkung des Magnetfeldes mit der Anisotropie der Magnetisierbarkeit in genau gleicher Weise verformt. Dieser Sachverhalt gilt auch dann, wenn die Linien sättigungsverbreitert sind und daher unterschiedliche Linienbreiten besitzen. In jedem Falle liefert also der Frequenzabstand zweier „korrespondierender" Stellen auf den Linienprofilen den $g_{J^{-}}$ Faktor. Bedeuten $v_{+}(\max )$ und $v_{-}(\max )$ die beim Felde $H$ gemessenen Frequenzen am Orte des Maximums von $I_{v}{ }^{+}$bzw. $I_{v}{ }^{-}$, so gilt

$$
v_{+}(\max )-v_{-}(\max )=-2 g_{J}\left(\mu_{n} / h\right) H,
$$

woraus wiederum durch Ausgleich und Mittelung der Bestwert $\bar{g}_{J}$ erhalten wird. Gleichung (14) bestimmt auch den kleinsten mit dem Spektrometer meßbaren $g_{J}$-Faktor, wenn maximal verfügbares Magnetfeld und Breite der „Linien“ $I_{v}{ }^{+}(v)$ und $I_{v}{ }^{-}(v)$ vorgegeben sind. - Zur Auswertung nach $\xi_{T}$ wird die Funktion $I_{v}{ }^{+}(v, H)\left[I_{\nu}{ }^{-}(v, H)\right]$ unter Vorgabe von $\Delta v$ (gemessen bei $\mathrm{H}=0), v_{0}, g_{J}=\bar{g}_{J}$ und eines geschätzten Wertes für $\xi_{T}$ in Abhängigkeit von $v$ in der Umgebung ihres Maximums für jede einzelne Feldstärke $H$ einer Meßreihe berechnet und die Frequenz $v_{+}(\max )_{\text {ber }}\left[\mathrm{bzw} . v_{-}(\max )_{\text {ber }}\right]$ ihres Maximums bestimmt. $\xi_{T}$ wird so lange variiert, bis eine im Mittel beste Anpassung der berechneten Frequenzen $v_{ \pm}(\max )_{\text {ber }}$ an die gemessenen $v_{ \pm}(\max )$ erreicht ist. Bei fast aufgelösten Linien ist beste Anpassung an die Form des registrierten Linienprofils ein zusätzliches Kriterium.

Eine Messung von $g_{J}$ und $\xi_{T}$ liefert nicht nur das „magnetische Dipolmoment" $g_{J} \mu_{n} J$ der Molekel im Rotationszustand $J$ und die Anisotropie der molaren Suszeptibilität

$$
\chi \perp-\chi \|=N_{\mathrm{A}}\left(\xi_{\perp}-\xi_{\|}\right)=3 N_{\mathrm{A}} \xi_{T}
$$

$\left(N_{\mathrm{A}}=\right.$ Avogadrosche $\left.\mathrm{Zahl}\right)$, sondern über die Interrelation ${ }^{6}$

$$
\frac{Q}{e}=\frac{I g_{J}}{M_{\mathrm{p}}}+\frac{12 m_{\mathrm{e}} c^{2}}{e^{2}} \xi_{T}
$$

auch das auf den Massenschwerpunkt bezogene Quadrupolmoment $Q$ der elektrischen Ladungsverteilung in der Molekel. Es bedeuten $e(>0)$ die Elementarladung, $I$ das molekulare Trägheitsmoment, $M_{\mathrm{p}}$ und $m_{\mathrm{e}}$ die Protonen- bzw. Elektronenmasse und $c$ die Lichtgeschwindigkeit. Für eine zweiatomige Molekel mit Kernen der Ladungszahlen $Z_{\mathrm{a}}$ und $Z_{\mathrm{b}}$ gilt insbesondere

$Q / e=\left(Z_{\mathrm{a}} z_{\mathrm{a}}{ }^{2}+Z_{\mathrm{b}} z_{\mathrm{b}}{ }^{2}\right)-\left\langle\sum_{i=1}^{Z_{\mathrm{a}}+Z_{\mathrm{b}}}\left(z_{i}{ }^{2}-x_{i}{ }^{2}\right)\right\rangle$.

$z$ und $x$ sind die Teilchenkoordinaten in einem molekelfesten $(x, y, z)$-Koordinatensystem mit Ursprung im Massenschwerpunkt und $z$-Achse parallel zur Kernverbindungslinie. $\left\langle\sum_{i}\left(z_{i}{ }^{2}-x_{i}{ }^{2}\right)\right\rangle$ ist ein $\mathrm{Ma} \beta$ für die Asymmetrie der Elektronenverteilung.

Die hier spektroskopierten Molekeln haben Kerne mit von Null verschiedenen Spins $I_{1}$ und $I_{2}$. Die resultierende Hyperfeinstrukturaufspaltung der Rotationslinien kann bis zum höchsten uns zugänglichen Magnetfeld vom Spektrometer nicht aufgelöst werden. Wir haben den Einfluß der Hyperfeinstruktur auf die Lage der Linien und die Form der gemessenen Linienprofile in Abhängigkeit vom Magnetfeld untersucht. Für die Cs-Halogenide ist nur die Hyperfeinstruktur infolge elektrischen Quadrupolmoments von Belang. In diesem Falle bleibt die zur Auswertung nach $g_{J}$ benutzte Gl. (14) gültig. Eine Korrektur für $\xi_{T} / h$ bleibt innerhalb der Meßunsicherheit. - Beim TlF dagegen ist die magnetische Hyerfeinstruktur infolge der Wechselwirkung des magnetischen Kerndipoloments mit dem von der Molekelrotation am Kernort erzeugten Magnetfeld und der Anisotropie der magnetischen Abschirmung (beides für den ${ }^{205} \mathrm{Tl}-\mathrm{Kern}$ ) so groß, daß unsere nach den Gleichungen für den kernspinlosen Fall ausgewerteten Meßergebnisse für $g_{J}$ und $\xi_{T} / h$ korrigiert werden müssen. Eine Rekonstruktion des Spektrums ohne und mit Hyperfeinstruktur (Molekelparameter aus ${ }^{7}$ ) ergibt, daß die Aufspaltung $v_{+}(\max )-v_{-}(\max )$ um $3,8 \%$ zu klein gemessen wird, d. h. der $g_{J}$-Faktor um 0,00020 vergrößert werden muß. Diese Korrektur ist nur wenig größer als die Meßunsicherheit. Der Einfluß der Hyperfeinstruktur auf die Bestimmung von $\xi_{T} / h$ wurde nur qualitativ untersucht mit dem Ergebnis, daß der Fehler kleiner als $10 \%$ sein muß. Unsere Bestimmung von $\xi_{T} / h$ hat einen Fehler von $17 \%$. Da wir $\xi_{T} / h$ nur aus der Linienschwerpunktsvariation bestimmen konnten und den etwaigen systematischen Fehler dieses Verfahrens nicht genau abschätzen können, verdoppeln wir das Fehlerintervall. 


\begin{tabular}{llllcll}
\hline Molekel & $\%$ & $I_{1}$ & $I_{2}$ & $J \rightarrow J+1$ & $v$ & ${ }^{\circ} \mathrm{C}$ \\
\hline${ }^{205} \mathrm{Tl}^{19} \mathrm{~F}$ & 70,5 & $1 / 2$ & $1 / 2$ & $1 \rightarrow 2$ & 0 & 400 \\
${ }^{133} \mathrm{Cs}^{19} \mathrm{~F}$ & 100 & $7 / 2$ & $1 / 2$ & $2 \rightarrow 3$ & $0,1,2,3,4$ & 600 \\
${ }^{133} \mathrm{Cs}^{35} \mathrm{Cl}$ & 75,4 & $7 / 2$ & $3 / 2$ & $5 \rightarrow 6$ & $0,1,2,3,4,5,6$ & 600 \\
${ }^{133} \mathrm{Cs}^{79} \mathrm{Br}$ & 50,5 & $7 / 2$ & $3 / 2$ & $11 \rightarrow 12$ & 0 & 600 \\
${ }^{133} \mathrm{Cs}^{127} \mathrm{I}$ & 100 & $7 / 2$ & $5 / 2$ & $17 \rightarrow 18$ & 0 & 600 \\
\hline
\end{tabular}

Tab. 1. Die spektroskopierten Molekeln, ihre (natürliche) Häufigkeit $(\%)$, die Spins $\left(I_{1}\right.$ und $\left.I_{2}\right)$ der beiden Kerne, die vermessenen Rotationsübergänge $(J \rightarrow J+1)$ bei den jeweiligen Schwingungszuständen $(v)$ und die ungefähre Temperatur $\left({ }^{\circ} \mathrm{C}\right)$ der Absorptionszelle.

\begin{tabular}{|c|c|c|c|c|c|c|c|c|c|}
\hline $\begin{array}{l}H \\
(\mathrm{kG})\end{array}$ & $\begin{array}{l}\widetilde{T} v \\
(\mathrm{kHz})\end{array}$ & $\begin{array}{l}v_{+}-v_{-} \\
(\mathrm{MHz})\end{array}$ & $g_{J}$ & $\begin{array}{l}\left\langle g_{J}\right. \\
\left(10^{-5}\right)\end{array}$ & $\begin{array}{l}\mathcal{J}_{1} \\
(\mathrm{kHz})\end{array}$ & 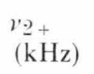 & $\stackrel{\nu_{2}}{(\mathrm{kHz})}$ & $\begin{array}{l}\triangle_{2+}+ \\
(\mathrm{kHz})\end{array}$ & $\begin{array}{l}\mathrm{d}_{2}- \\
(\mathrm{kHz})\end{array}$ \\
\hline 16.09 & 200 & 1.306 & 0.05324 & 81.5 & 6 & 180 & 187 & 32 & 25 \\
\hline 17,86 & 200 & 1,445 & 0,05307 & 73,5 & 12 & 207 & 219 & 1 & -11 \\
\hline 19.66 & 250 & 1,585 & 0,05288 & 83,4 & 19 & 189 & 207 & 15 & -3 \\
\hline 22,19 & 250 & 1,802 & 0,05327 & 73,9 & 8 & 210 & 219 & -14 & -23 \\
\hline 24.75 & 290 & 1.998 & 0.05295 & 76,9 & 21 & 173 & 194 & 13 & -8 \\
\hline 26,55 & 300 & 2,186 & 0,05401 & 74,1 & -21 & 195 & 175 & -19 & 1 \\
\hline 29,47 & 300 & 2,410 & 0,05364 & 66,8 & -6 & 161 & 155 & -1 & 5 \\
\hline 31,38 & 300 & 2,546 & 0,05322 & 62,7 & 13 & 168 & 182 & -20 & -32 \\
\hline 36,51 & 350 & 2,984 & 0,05361 & 62,9 & -6 & 152 & 145 & -36 & -29 \\
\hline 38,33 & 350 & 3,163 & 0.05413 & 59,9 & -37 & 129 & 92 & -23 & 14 \\
\hline 40,74 & 350 & 3,341 & 0,05379 & 56,3 & -18 & 92 & 73 & -4 & 15 \\
\hline
\end{tabular}

Tab. 2. Meßreihe zur Auswertung nach $g_{J}$ und $\xi_{T} / h$ für ${ }^{205} \mathrm{Tl}^{19} \mathrm{~F}$.

$v_{ \pm}=v_{ \pm}(\max ) ;$

$v_{2}=v_{ \pm} \pm \bar{g}_{J}\left(\mu_{n} / h\right) H$ $-26669,000 \mathrm{MHz}$;

$\Delta_{1}=2\left|\bar{g}_{J}\right|\left(\mu_{n} / h\right) H$ $-\left(v_{+}-v_{-}\right)$und

$\Lambda_{2 \pm}=v_{ \pm}(\max )$ ber $-v_{ \pm}(\max )$ sind die Differenzen zwischen berechneten und gemessenen Frequenzen.
Tab. 3. $g_{J}$-Faktor und Anisotropie der Magnetisierbarkeit.

\begin{tabular}{|c|c|c|c|}
\hline Molekel & $v$ & $\left|g_{J}\right|$ & $\begin{array}{l}\xi_{T} / h \\
{\left[\mathrm{~Hz} /(\mathrm{kG})^{2}\right]}\end{array}$ \\
\hline \multirow[t]{2}{*}{${ }^{205} \mathrm{Tl}^{19} \mathrm{~F}$} & 0 & $0,05370(15)$ & $600(200)$ \\
\hline & & $0,053530(40)(a)$ & $450(200)(\mathrm{b})$ \\
\hline \multirow[t]{6}{*}{${ }^{133} \mathrm{Cs}^{19} \mathrm{~F}$} & 0 & $0,06413(18)$ & $805(60)$ \\
\hline & & $\begin{array}{l}0,064200(26)(\mathrm{c}) \\
0.0621(55)(\mathrm{d})\end{array}$ & $740(300)(\mathrm{c})$ \\
\hline & 1 & $\begin{array}{l}0,06444(30) \\
0,063939(52)(\mathrm{c})\end{array}$ & $880(150)$ \\
\hline & 2 & $0,06384(22)$ & $860(200)$ \\
\hline & 3 & $0,06355(21)$ & $735(150)$ \\
\hline & 4 & $0,06421(88)$ & $750(300)$ \\
\hline \multirow[t]{8}{*}{${ }^{133} \mathrm{Cs}^{35} \mathrm{Cl}$} & 0 & $0,02803(7)$ & $1200(200)$ \\
\hline & & $0,0212(11)(d)$ & \\
\hline & 1 & $0,02756(17)$ & - \\
\hline & 2 & $0,02699(25)$ & - \\
\hline & 3 & $0,02699(23)$ & - \\
\hline & 4 & $0,02669(26)$ & - \\
\hline & 5 & $0,02648(28)$ & - \\
\hline & 6 & $0,02630(23)$ & - \\
\hline${ }^{133} \mathrm{Cs} \mathrm{s}^{79} \mathrm{Br}$ & 0 & $0,0099(10)$ & - \\
\hline${ }^{133} \mathrm{Css}^{127} \mathrm{I}$ & 0 & $<0,0036$ & - \\
\hline
\end{tabular}

(a) W. Drechsler u. G. Gräff, Z. Physik 163, 165 [1961].

(b) R. von Boeckh, G. Gräff u. R. Ley, Z. Physik 179, 285 [1964].

(c) G. GräfF u. Ö. Runólfsson, Z. Physik 187, 140 [1965].

(d) F. Mehran, R. A. Brooks u. N. F. Ramsey. Phys. Rev. 141, 93 [1966].

Mit dem beschriebenen Spektrometer wurden die in Tab. 1 aufgeführten Molekeln untersucht. Aus Intensitätsgründen wurde die jeweils häufigste Isotopenkombination ausgewählt. In Tab. 2 ist als Beispiel eine Meßreihe zur Auswertung nach $g_{J}$ und
Tab. 4. Mit den Bestwerten von $g_{J}$ und $\xi_{T} / h$ für $v=0$ berechnete Molekelkonstanten: Anisotropie $\chi_{\perp}-\chi_{\|}$der molaren Suszeptibilität, auf den Massenschwerpunkt bezogenes Quadrupolmoment $Q / e$ der elektrischen Ladungsverteilung und Asymmetrie $\left\langle\Sigma\left(z i^{2}-x_{i}{ }^{2}\right)\right\rangle$ der Elektronenverteilung in der Molekel.

\begin{tabular}{lclr}
\hline Molekel & $\begin{array}{l}\chi_{1}^{-6} \chi_{\|} \\
{\left[10^{-6} \mathrm{erg} /\left(\mathrm{G}^{2} \mathrm{~mol}\right)\right]}\end{array}$ & $\begin{array}{l}Q / e \\
{\left[\AA^{2}\right]}\end{array}$ & $\left\langle\sum_{\left[\AA^{2}\right]}\left(z_{i}{ }^{2}-x_{i}^{2}\right)\right\rangle$ \\
\hline${ }^{205} \mathrm{Tl}^{19} \mathrm{~F}$ & $7,2(24)$ & $-2,34(57)$ & $37,76(57)$ \\
${ }^{133} \mathrm{Cs}^{19} \mathrm{~F}$ & $9,64(72)$ & $-3,57(17)$ & $46,37(17)$ \\
${ }^{133} \mathrm{Cs}^{35} \mathrm{Cl}$ & $14,4(24)$ & $-3,15(58)$ & $113,89(58)$ \\
\hline
\end{tabular}

$\xi_{T} / h$ für die ${ }^{205} \mathrm{Tl}^{19} \mathrm{~F}$-Molekel dargestellt. Tabelle 3 enthält unsere Meßergebnisse und zum Vergleich diejenigen anderer Autoren. Mit den Bestwerten von $g_{J}$ und $\xi_{T} / h$ berechnete Molekelkonstanten [Gln. (15), (16) und (17)] sind in Tab. 4 zusammengestellt.

Die Vorzeichen der $g_{J}$-Faktoren haben wir nicht bestimmt; sie sind zweifelsfrei sämtlich negativ. Zur Berechnung des $g_{J}$-Faktors wurde $\mu_{n} / h=0,76227 \mathrm{MHz} /$ kG benutzt. Der angegebene Wert für $\left|g_{J}\right|$ ist der nach der Methode der kleinsten Fehlerquadrate bestimmte Mittelwert aus den Einzelmessungen bei verschiedenen Feldstärken unter Berücksichtigung des Gewichtes $p$ der Einzelmessung. $2 \widetilde{\Delta v}(H)$ sei die volle Halbwertsbreite einer unaufgelösten Liniengruppe. Bei ausreichendem Signal - Rausch-Verhältnis wird (1/10) $\widetilde{\Delta v}(H)$ als Ableseunsicherheit der Linienfrequenzen $v_{+}(\max )$ angenommen. Dann ist der Ablesefehler einer Einzel- 
messung von $g_{J}$

$$
\Delta g_{J}=\frac{(1 / 10) \widetilde{\Delta v}(H)}{v_{+}(\max )-v_{-}(\max )} g_{J}
$$

und $p \sim 1 /\left(\Delta g_{J}\right)^{2}$ das Gewicht der Einzelmessung. $\overline{\Delta g_{J}}$ sei das arithmetische Mittel der Ablesefahler von $g_{J}, n$ die Anzahl der Einzelmessungen und $\sigma_{m}$ die Standardabweichung des Mittelwertes von $g_{J}$. Der in Einheiten der letzten Stelle angegebene Fehler von $g_{J}$ ist $\sigma_{m}$, wenn $\overline{\Delta g_{J}} / \sqrt{n}$ und $\sigma_{m}$ von annähernd gleicher Größe sind. Übersteigt $\overline{\Delta g_{J}} / \sqrt{n}$ den statistischen Fehler $\sigma_{m}$ um mehr als das dreifache, so ist $\overline{\Delta g_{J}} / V_{n}$ notiert.

Bei dem beschriebenen Anpaßverfahren zur Auswertung nach $\xi_{T} / h$ wurden die Einzelmessungen entsprechend mit

$$
p^{\prime} \sim\left[\frac{v_{ \pm}(\max )-v_{0} \pm \bar{g}_{J}\left(\mu_{n} / h\right) H}{(1 / 10) \widetilde{\Delta} v(H)}\right]^{2}
$$

gewogen. Eine zu $g_{J}$ analoge Fehlerrechnung ist für $\xi_{T} / h$ nicht angebracht, da hier durch Variation von $v_{0}$ und $\Delta v$ in $\mathrm{Gl}$. (10) weit größere Fehlergrenzen resultieren. Deshalb wird in diesem Falle von der Unsicherheit in $v_{0}( \pm 10 \mathrm{kHz})$ und $\Delta v( \pm 50 \mathrm{kHz})$ ausgegangen und die hieraus folgende Schwankungsbreite des ausgeglichenen Wertes für $\xi_{T} / h$ als Fehler angegeben.

Die von uns gemessenen $g_{J}$ Faktoren der Molekeln TIF, CsF und $\mathrm{CsCl}$ im Schwingungsgrundzustand $(v=0)$ sind auf etwa $3 \%$ genau. $g_{J}(\mathrm{TIF})$ und $g_{J}(\mathrm{CsF})$ stimmen mit den von GRÄFF u. a. ${ }^{1}$ gemessenen Werten überein. Keine Übereinstimmung besteht mit dem von RAMSEY u. a. ${ }^{2}$ angegebenen $g_{J^{-}}$ Faktor (,$g_{J \text { (corrected) }}$ ) ) für ${ }^{133} \mathrm{Cs}^{35} \mathrm{Cl}$; dagegen ist die Abweichung von RAMSEY's nicht korrigiertem Meßwert $, g_{\text {exp peak }}{ }^{\prime}=-0,026698(71) \quad$ geringer. Wir haben den $g_{J}$-Faktor für die Molekeln CsF und $\mathrm{CsCl}$ auch in höheren Schwingungszuständen $(v>0)$ gemessen. Beim $\mathrm{CsF}$ sind die Fehlergrenzen noch zu groß, um eine Variation von $g_{J}$ mit $v$ quantitativ zu formulieren. Dagegen finden wir für ${ }^{133} \mathrm{Cs}^{35} \mathrm{Cl}$ :

$g_{J}(v)=-0,02815(7)+0,00031(8)(v+1 / 2)$.

Bei den Messungen am CsBr ergaben sich Schwierigkeiten mit der Stark-Modulation. Es war nicht mög-

1 TIF: W. Drechsler u. G. GräfF, Z. Physik 163, 165 [1961], CsF: G. GräfF u. Ö. Runólfsson, Z. Physik 187, 140 [1965].

2 F. Mehran, R. A. Brooks u. N. F. Ramsey, Phys. Rev. 141, 93 [1966].

3 W. Hüttner u. W. H. Flygare, J. Chem. Phys. 47, 4137 [1967]. Obgleich Magnetfelder bis fast $50 \mathrm{kG}$ angelegt werden, braucht in Gl. (1) ein weiteres Glied proportional zu $H^{3}$ noch nicht berücksichtigt zu werden. Vgl. hierzu: W. H. Flygare u. R. C. Benson, Mol. Phys. 20, 225 [1971].

4 C. H. Townes u. A. L. SCHawlow, Microwave Spectroscopy, McGraw-Hill Book Co., New York 1955, S. 23.

5 Bei hinreichend kleinem $H$-Feld werden sämtliche $2 J+1$ $\sigma$-Komponenten einer jeden Gruppe $m \rightarrow m+1$ und $m \rightarrow$ $m-1$ im Vergleich zu ihrer Linienbreite nur wenig unter- lich, die Rotationslinie $J=11 \rightarrow 12$ bei einem Magnetfeld von $45 \mathrm{kG}$ durch Stark-Effekt zu modulieren, da schon bei verhältnismäßig kleinen Stark-Spannungen eine Gasentladung in der Zelle einsetzte. Die Linie wurde deshalb mit frequenzmodulierter Klystronstrahlung nachgewiesen (wodurch allerdings ein Verlust an Empfindlichkeit um etwa den Faktor 10 in Kauf genommen werde mußte). Die Modulationsfrequenz betrug 1/3 der Empfangsfrequenz $(100 \mathrm{kHz})$ des phasenempfindlichen Gleichrichters. Die beiden Liniengruppen $m \rightarrow m+1$ und $m \rightarrow m-1$ spalten selbst bei einer Feldstärke von $45 \mathrm{kG}$ noch nicht vollständig auf. Unser in Tab. 3 angegebener Meßwert für $g_{J}$ ist mit einem größeren Fehler behaftet, weil die Messung des Frequenzabstandes $v_{+}(\max )-v_{-}(\max )$ bei frequenzmodulierter Strahlung nur mit geringerer Genauigkeit möglich war. Wir haben versucht, eine Aufspaltung der Linie $J=17 \rightarrow 18$ der ${ }^{133} \mathrm{Cs}^{127}$ I-Molekel bei $44 \mathrm{kG}$ zu messen. Es wurde keine Einsattelung, geschweige denn eine Aufspaltung, sondern nur eine Verbreiterung der Linie um etwa den Faktor 4/3 beobachtet. Hieraus errechnet man die in Tab. 3 verzeichnete obere Schranke für $\left|g_{J}\right|$.

Unsere Meßwerte für die magnetische Anisotropie $\xi_{T} / h$ stimmen ebenfalls mit denen von GRÄFF u. a. ${ }^{7,1}$ überein. Beim CsF im Schwingungsgrundzustand deutet sich bei hohen Magnetfeldern eine Auflösung der Summenlinien nach der $m$-Unterstruktur an. Durch diese zusätzliche Information im registrierten Linienprofil konnte der Fehler unseres $\xi_{T} / h$-Wertes merklich verringert werden.

Wir danken Herrn Dr. M. Plato für Hilfe bei der Aufstellung einiger Rechenprogramme, den Herren Prof. Dr. T. Törring und K. Pietraszyk für die Bereitstellung des Stark-Generators und dem Leiter der Institutswerkstatt, Herrn H. HAHNe, sowie seinem Mitarbeiter, Herrn F. BÖRNER, für Beratung beim Bau des Spektrometers. Unser Dank gilt der Deutschen Forschungsgemeinschaft für die großzügige finanzielle Unterstützung dieser Arbeit.

schiedlich verschoben, so daß das Anisotropieglied nur eine Verschiebung des jeweiligen Linienschwerpunktes $v_{ \pm}(S)$ bewirkt. Es gilt

$v_{ \pm}(S)=v_{0} \mp g_{J}\left(\mu_{n} / h\right) H-\left(\xi_{T} / h\right)-3 \frac{J+1}{5} \frac{3}{(2 J+1)(2 J+3)} H^{2}$,

d. h. für höhere $J$-Werte nimmt diese Verschiebung wie $1 / J$ ab.

6 W. Gordy u. R. L. Cook, Microwave Molecular Spectra, John Wiley \& Sons, New York 1970, S. 396. Über die Problematik der Anwendbarkeit von Gl. (16) auf schwere Molekeln (TlCl) vgl. R. Ley u. W. Schauer, Z. Naturforsch. 27 a, 77 [1972].

7 R. von Boeckh, G. Gräff u. R. Ley, Z. Physik 179, 285 [1964]. 